\title{
Los procesos migratorios como expresión de integración territorial de México y Centroamérica
}

\author{
Antonio Mosquera Aguilar \\ Universidad Autónoma de Chiapas
}

\begin{abstract}
Resumen
Este documento presenta algunas consideraciones acerca de la migración guatemalteca a México como expresión demográfica de un proceso de integración, en todos los planos de la realidad, entre dos países vecinos. Cuando el flujo migratorio ha crecido en magnitud adquiriendo un carácter de movimiento de masas, se enfrenta con dificultades en su conceptualización. Esta migración no es un proceso que enfrente a dos poblaciones con características culturales diferentes, sino que se trata de un mayor grado de relaciones entre las poblaciones de Guatemala y el estado mexicano de Chiapas, donde sus habitantes poseen en muchos sentidos, rasgos culturales iguales y se enfrentan, en su desarrollo social a contradicciones sociales similares. Después de conocer las corrientes migratorias se sitúa el ámbito del problema haciendo una regionalización que ubique geográficamente el fenómeno. La aceptación de las regiones fronterizas que se proponen en este estudio será de utilidad para el acopio futuro de datos.
\end{abstract}

Palabras clave: migración, frontera Guatemala-Chiapas, grupos indígenas mayas, desplazamiento, refugiados.

\begin{abstract}
This document offers some considerations regarding Guatemalan migration to Mexico as the demographic expression of an integration process between two neighbor countries at all levels of reality. When the migratory flow has grown and acquires the character of mass movement, it starts facing difficulties to be conceptualized. This migration is not the kind of process that confronts two peoples with different cultural characteristics; on the contrary, it refers to two communities with a higher degree of relations which are located between Guatemala and the Mexican state of Chiapas, where many of their inhabitants have the same cultural characteristics in many senses, and they face similar contradictions for their social development. Once the migratory currents are recognized, the background of this problem is defined through a regionalization to geographically locate the phenomenon. If the border regions proposed in this research are accepted, these tools will be useful for future gathering of data.
\end{abstract}

Keywords: migration, Guatemala-Chiapas border, Mayan Indian groups, displacement, refugees. 


\title{
LOS PROCESOS MIGRATORIOS COMO EXPRESION DE LA INTEGRACION TERRITORIAL DE MEXICO Y CENTROAMERICA
}

\author{
Por \\ Antonio Mosquera Aguilar*
}

\section{ALGUNAS CONSIDERACIONES ACERCA DE LA MIGRACION GUATEMALTECA A MEXICO}

La migración guatemalteca a México es la expresión demográfica de un proceso de integración, en todos lo planos de la realidad, entre dos países vecinos. Cuando su flujo ha crecido en magnitud y ha adquirido carácter de movimiento de masas, se ha enfrentado con dificultades en su conceptualización. El debate ha puesto a prueba los límites del sistema político mexicano así como ha demostrado la incapacidad de creación de política de la oligarquía militarizada guatemalteca.

Esta migración no es un proceso que enfrente a dos poblaciones con características culturales diferentes, sino se trata de un mayor grado de relaciones entre las poblaciones de Guatemala y el estado mexicano de Chiapas, donde sus habitantes poseen en muchos sentidos, rasgos culturales iguales y se enfrentan, en su desarrollo social, a contradicciones sociales similares.

La migración internacional actual no es motivo de mucha preocupación en los estudios demográficos, tal vez porque afecta a una población relativamente pequeña en relación con la mundial; según Pierre Georgs no pasa del dos o tres por ciento (Tabbarah, 1982).

Sin embargo, en México la migración internacional ha sido objeto de gran atención a partir de la década de los años setenta. El centro de atención ha sido invariablemente la migración mexicana hacia los Estados Unidos de América (EUA). En gran medida, esta

- Maestro en sociología del programa Pontificia Universidad Católica de Ecuador y Consejo Latinoamericano de Ciencias Sociales PUECE*CLACSO, actualmente profesor del área de Ciencias Sociales de la Universidad Autónoma de Chiapas en la carrera de Sociología. 
preocupación se debe a que EUA decidió imponer restricciones a la migración mexicana en 1963 y 1964, de acuerdo a la tradición según la cual "los trabajadores mexicanos han sido cortejados en las épocas de escasez de mano de obra, pero han sido tratados como extranjeros indeseables cuando la escasez desaparece". (Blejer, et al. 1982). Al convertirse en problemática la relación México-EUA en este aspecto, estudiosos de ambos países han intentado comprender el fenómeno para poder orientar la política de sus respectivas naciones. No está de más indicar que el punto de debate está constituido por la importancia de la población indocumentada, es aquí donde las autoridades de EUA tienen una posición altamente ideologizada (Tuirán, 1983).

La dificultades de la migración mexicana hacia EUAdemuestran un cambio en las características de la migración internacionalmoderna. En efecto, durante el siglo pasado fue una constante la ocupación de los "espacios vacíos" (EUA, Argentina, Australia, Nueva Zelanda, Canadá, etc.), pero en la actualidad hay restricciones a la movilización internacional que tiene nuevas características: primero, los países expulsores son en su mayoría los menos desarrollados; segundo, ocurre el drenaje de cerebros, al emigrar trabajadores altamente calificados; tercero, se incrementa la migración indocumentada especialmente de trabajadores agrícolas o no calificados; cuarto, aparece la migración interregional entre países en desarrollo; y por último, se manifiestan flujos de "migrantes forzados" a causa de guerras, persecusión o desastre natural, tal como lo resume Riad Tabbarah (Tabbarah, 1982: 118-119).

Estas notas distintivas que Tabbarah ha resumido para el mundo se refieren a un proceso actual, de aquí que, por ser algo nuevo, resulte difícil percibirlo. Así, en México, el conocimiento de las difucultades de la migración hacia EUA surge en forma generalizada, cuando el fenómeno ha adquirido repercusión importante y ha vuelto problemáticas las relaciones internacionales.

Conviene hacer esta mención puesto que los enfoques teóricos hechos para la frontera norte de México influenciaron los acercamientos explicativos sobre la migración guatemaltca a México (Wasserstrom, 1976; Pohlenz y Castillo, 1982; Pohlenz, 1978; Velasco, 1979). En gran medida era un traslado de los temas, una repetición estereotipada de condenas al capitalismo odolientes quejas porque 
"las fincas prefieren a trabajadores guatemaltecos y no a mexicanos, ya que los primeros aceptan peores condiciones y salarios".

A lo dicho hay que añadir otra consideración que se hizo presente en cl análisis de la migración guatemalteca, formada en torno a la institución del derecho de asilo que surge en latinoamérica después de la lucha de independencia, buscando dar lugar a la libre expresión de ideas, al garantizar a los políticos una protección segura a su vida y libertad en las legaciones latinoamericanas, así comolaimposibilidad de otorgar extradiciones. El derecho internacional latinoamericano ha regulado desde 1865 el asilo, dando lugar a una importante reflexión sobre su carácter humanitario. ${ }^{1}$

Sabido es que México recibió un importante flujo de exiliados por la guerra civil española de los años treinta de este siglo, igualmente, la embajada mexicana fue buscada en los casos de derrocamiento de los gobiernos constitucionales de Guatemala en 1954 y Chile en 1973; hechos que tuvieron gran repercusión por tratarse de los primeros casos de asilo diplomático masivo. Sin ambargo, lo aparatoso de tales ingresos no ticne significación demográfica de importancia.

En 1930 comienza a definirse una política de población explícita con la Ley de Migración expedida a iniciativa del Presidente Pascual Ortiz Rubio, donde se señalaba el fenómeno de la inmigración como atribuciones de la Secretaría de Gobernación. Tal regulación fue recogida en el artículo 4 de la Ley General de Pobiación promulgada por el Presidente Lázaro Cardenas en 1936. La nueva Ley General de Población de 1947 repite tal disposición (Mora Bravo 1984: 93). A pesar de tales pronunciamientos formales, nunca se ejecutaron cstas disposiciones con liberalidad, hecho comprobable, pues los nacionales extranjeros en México que provienen principalmente de EUA y Centroamérica, y de manera secundaria de Europa y otras áreas, han disminuido en términos relativos durante la vigencia de tal política. De esta manera, los extranjeros eran menos del $1 \%$ en 1930 y en 1960, y para 1970 representaba apenas el $0.5 \%$ de la población total (Alba 1984: 57-58).

${ }^{1}$ México ha firmado la Convención sobre Asilo de 1928, la Convención sobre Asilo Político de 1933, la Convención sobre Asilo Diplomático de 1954. Véase Barrios Ruiz (1976). 
Cabe destacar que la legislación sobre inmigración y el derecho de asilo no se contradecían; no importaba que hubiera una práctica restrictiva, puestoda expresión pública se ajusta a tener por bienvenida a la inmigración, porque ayudaba a incrementar la población. Esta situación cambió a partir de la nueva Ley General de Población publicada en 1976, que suprimió la disposición que ordenaba alentar la inmigración y significó la culminación de un alargado debate. Desde esa fecha se ha señalado por diferentes funcionarios la necesidad de controlar o disminuir la tasa de crecimiento de la población. ${ }^{2}$ Uno de los elementos que contribuyen a la formación de dicha tasa es la inmigración, de donde resulta que ésta debe ser normada para hacer más fácil el descenso de aquélla.

El nudo de la polémica teórica alrededor de la migración guatemalteca consistió en que, desde su inicio, se conceptualizó ideológicamente: se confunden las apreciaciones jurídicas con hechos reales. Al integrarse los elementos que se han señalado resulta que la migración guatemalteca es una corriente equivalente a la migración mexicana a EUA, pero a diferencia de ésta, que es beneficiosa para el crecimiento del capital, la guatemalteca es una carga adicional a los esfucrzos de México para desarrollarse, pues todo crecimiento de la población se define como negativo a partir de las definiciones de política demográfica concretada en la Ley General de Población, lo que conduce a una postura restrictiva en el otorgamiento de asilo a los guatemaltecos. Los elementos estaban presentes pero faltaba el portavoz que los integrase y que a la vez tuviera suficiente poder como para que su voz fuera escuchada.

La directora de servicios migratorios, Diana Torres Arciniega fue quien abrió la polémica al describir la migración guatemalteca. Se ser̃ala a Torres Arciniega no porque su punto de vista haya sido original, pues como consta tenía suficientes antecedentes, sino porque su posición hacía valederos los criterios que sostenía. Se insiste en que se trata de una posicion ideológica: seguramente no revisó lo existente a nivel bibliográfico ni ordenó una investigación sobre el

${ }^{2}$ Mora Bravo (1984: 126 y 55). Tanto el presidente Luis Echeverría Alvarez como José López Portillo manifestaron la necesidad de disminuir esta tasa. El presidente Miguel de la Madrid, además de reconocer la continuidad de esta política, adicionó objetivos cualitativos. 
problema, sólo realizó la integración de los elementos señalados que coincidían con definiciones políticas que se esforzaban en seguir intereses atribuibles socialmente.

La funcionaria consideraba que el migrante podía ser econónico o político. El "económico" viajaba a México en busca de "residencia y progreso" y debidoa la demanda de servicios así como a los problemas sociales que causaba, era una corriente indeseable. Es evidente que la búsqueda de residencia y progreso es connatural al hombre tanto como el derecho al trabajo, de donde se infiere que toda persona es "cconómica", por tanto, es claroque la funcionaria deseaba señalar que cl ingreso de tales migrantes era de carácter restrictivo. Pero había una excepción a la regla, el migrante político podía ser aceptado, pues no se había renunciado a la tradición latinoamericana del asilo. Los políticos eran los que se encontraban en "el sitio de lucha" según Torres Arciniega, en consecuencia al abandonarlo, se convierten en "cconómicos", si atendemos al razonamiento lógico. Así, bajo el criterio de la funcionaria, el migrante político era una contradicción en sus términos y por lo tanto se tornaba en una categoría imposible. ${ }^{3}$

Frente a estos despropósitos, hubo quien intentó mantener la idea de la tipología para darle normatividad a las decisiones migratorias. El problema no residía en mantener una política de inmigración restrictiva, sino en que los mismos migrantes guatemaltecos tenían un "perfil borroso"; con ellos se vuelve más borrosa la frontera móvil entre asilado, refugiado, desplazadoo "migrante económico" (Aguayo, 1984: 17). Una vez que existieran criterios precisos, se podría orientar la acción gubernamental al reconocer la confusa categoría de refugiado; de este modo se precisaría a los trabajadores que pueden deportarse sin problemas. Esta posición no rompe con la dicotomía de migrante económico y migrante político, sino solamente intenta definir al último para impedir el uso ilimitado de la autoridad soberana; vale decir, intenta limitar el poder discrecional del Ejecutivo para proteger a determinadas personas por encima de intereses políticos $y$ diplomáticos.

${ }^{3}$ Los conceptos de la licenciada Diana Torres Arciniega, directora general de Servicios Migratorios, fueron explícitos en su intervención en la mesa redonda sobre "Corrientes migratorias centroamericanas", organizada en Tuxtla Gutiérrez, Chiapas, el 21 de mayo de 1982. Existe documento mimeografiado de la misma. 
Fuera de las posiciones que se encaminan a dar racionalidad a las medidas restrictivas de la inmigración, cuyo más destacado expositor es Sergio Aguayo Quezada (1984; 1985a; 1985b), hay opositores más tenaces. Aunque ha habido cambios en las autoridades migratorias a partir de 1983, éstas han continuado la aplicación de los mismos criterios políticos. El debate con estos funcionarios no se puede calificar de tranquilo, antes bien, al haberse recurrido a medidas administrativas abusivas que intentaron silenciarlo, se convirtió en escabroso.

Desde 1981, varios sectores de la población mexicana expresaron su oposición a la limitación de la inmigración guatemalteca y controamericana. Entre estos sectores destacaron las voces de la Diócesis de San Cristobal de Las Casas bajo la autoridad del obispo católico Samuel Ruiz y la del escritor Adolfo Aguilar Sinzers; en general, sostenían que no debía haber restricciones a la migración guatemalteca y centroamericana. Tal oposición se intentó silenciar en julio de 1984, al detenerse a un sacerdote, dos religiosas y una enfermera relacionados con la mencionada Diócesis y en hecho aparte, también al escritor Aquilar Sínzer (Mayorga, 1984). Algunos optaron por señalar que la corriente guatemalteca a México no tenía un número significativo como para tomarla en cuenta en un análisis migratorio ${ }^{6}$. No obstante, el resultado de estos excesos arbitrarios fue interesar a un mayor número de gente en el conocimiento del fenómeno, entre las que cabe destacar, entre otras, la consulta popular que organizó el Senado ${ }^{7}$.Aunque ha crecido elinterésy debate público, no ha habido cambios en la política de población: hasta ahora la migración guatemalteca a México no se considera deseable.

${ }^{4}$ La posición de la Diócesis consta en el boletín que se edita bajo el título de "Comité Cristiano de Solidaridad de la Diócesis de San Cristobal de Las Casas".

5 Las opiniones del escritor han sido constantemente expresadas en conferencias y artículos periodísticos (véase los artículos de Aguilar Sínzer en Nexos No.55, y el periódico unomásuno de los días 9 de mayo, 6 y 9 de junio, 14 y 29 de julio y 4 de agosto de 1984).

${ }^{6}$ Posición sostenida por el ganador del Premio de la Academia de Investigación Científica correspondiente a Ciencias Sociales, Doctor Humberto Muñoz García, quien ha realizado investigaciones sobre procesos migratorios desde 1965. (Véase: "Es đifícil saber lo que incidió la crisis económica en el fenómeno migratorio: Humberto Muñoz García" por Rocío Incera/I en unomásuno del 5 de diciembre de 1984) p.15.

${ }^{7}$ La consulta popular fue inaugurada por el Senado para conocer la magnitud del fenómeno migratorio mexicano. Cfr."Consulta popular del Senado sobre trabajađores migratorios" en unomásuno (México, D. F., 13 de marzo de 1985); este foro popular fue resultado de una gira de trabajo realizada por la Comisión de la Frontera Sur del Senado de la República un mes antes. Cfr. "Supervisan legisladores en 4 estados el 
A pesar de que la búsqueda por conceptualizar la migración guatemalteca a México no cuenta con ningún aporte del país de origen, va en aumento el reconocimiento de este fenómeno. Tal situación se puede comprobar en la declaraciones del Secretario General del Consejo Nacional de Población, Gerónimo Martínez García, en el sentido de que el número de refugiados no afecta las metas poblacionales, y que existe conocimiento de otras corrientes migratorias de guatemaltecos ${ }^{8}$.

Los acercamientos de campo permiten distinguir las siguientes corrientes: 1) Una estacional, con destino a las fincas de café y caña de azúcar cercanas a la frontera; a) Una permanente, con destino a fincas de caña de azúcar y banano del Soconusco; 3) Una forzada, con destino a campos de refugiados; y4) otras consistentes en migraciones permanentes por diversos motivos (familiares, negocios, trabajos varios), así como de migrantes con destino a EUA.

\section{LAS REGIONES FRONTERIZAS DE GUATEMALA Y CHIAPAS}

La existencia de diversas interrelaciones que se caracterizan por su alta intensidad y se concretan en una continuidad especial entre México y Guatemala, se expresan en varias regiones que podemos calificar de fronterizas. El manejo regional a nivel teórico

avance logrado por la Comisión de la Frontera Sur" por Rigoberto Santiago/Juan Guzmán en unomásuno (México, D. F., 9 de febrero de 1985); el foro realizado en San Cristóbal de Las Casas del 6 al 8 de mayo đe 1985, recibió 12 ponencias sobre la situación de la frontera sur. Cfr. "Los emigrados de Guatemala, grave problema en Chiapas" por Rosa Rojas en La jornada (México, D. F. 9 de mayo de 1985); en la audiencia pública celebrada en el campamento Los Lirios de Quintana Roo, se escuchó a refugiados. Cfr. "México no cerrará puertas ni expulsará refugiados" por Jeanette Becerra Acosta en unomásuno (México, D. F., 16 de junio de 1985) y "México es intransigente y celoso en la solidaridad con Centroamérica: senadores" por Jeanette Becerra Acosta en unomásuno (México, D.F., 17 de junio de 1986). El programa cultural de las fronteras de la Secretaría de Educación Pública se vio fortalecido por el Convenio de Coordinación y Colaboración Cultural que aunó esfuerzos con los estados de Campeche, Chiapas, Quintana Roo, Tabasco y Yucatán. Cfr. Integración (México, D. F., año I, Vol.1, Nov.-Dic. 1984). El convenio firmađo en 1984 permite la organización de foros para tratar las relaciones México-Centroamérica.

8 Véase: "El número de refugiados no altera el programa poblacional: el CONAPO" por Rodrigo Huerta en unomásuno (México, D.F., 19 de febrero de 1985) yGerónimo Martínez García, "La política de población de México". Conferencia dictada por el secretario general del Consejo Nacional de Población, en el Centro de Capacitación para el Desarrollo de la Secretaría de Programación y Presupuesto. México, D. F., 16 de mayo de 1985, fotocopia. pp. 13 a 15. 
permite tener una perspectiva homogénea de los lugares de destino y expulsión que caracterizan los procesos migratorios (véase: Mosquera Aquilar, 1978: 1-7).

\section{Definición de las regiones fronterizas de Chiapas}

Partiendo de reconocer la existencia de la Sierra Madre de Chiapas como una frontera natural imponente, se puede señalar que una región esta constituida por la vertiente hacia el pacífico, lo que coincide con la mayoría de los autores; el antiplano de la misma Sierra Madre, se identifica como otra región; la depresión central de Chiapas también hace frontera con Guatemala y por último, la selva lacandona participa de las mismas catacterísticas. Así, sólo nos resta identificar los municipios que están afectados de fuerte migración guatemalteca y participan de la calidad de fronterizos. A continuación hacemos un listado de las regiones y los respectivos municipios que las integran:

CUADRO 1. Municipios que integran las regiones fronterizas de Chiapas.

\section{REGION SOCONUSCO \\ Municipios}

001 Acacoyagua

003 Acapetagua

015 Cacahoatán

032 Escuintla

035 Frontera Hidalgo

037 Huehuetán

040 Huixtla

051 Mapastepec

054 Mazatán

055 Metapa

071 Pueblo Nuevo Comaltitlán

087 Suchiate

089 Tapachula

102 Tuxtla Chico

103 Tuzantán

105 Unión Juárez

Total de la Región
Extensión en $\mathrm{km}^{2}$

191.30

658.30

173.90

206.20

106.80

313.00

385.00

1085.60

382.60

101.80

606.10

303.00

857.00

64.60

268.30

72.00

5775.50 


\begin{tabular}{llr}
$\begin{array}{l}\text { REGION SIERRA } \\
\text { Municipios }\end{array}$ & Extensión en $\mathrm{km}^{2}$ \\
\hline 010 & Bejucal de Ocampo & 82.00 \\
011 & Bella Vista & 114.30 \\
036 & La Grandeza & 52.20 \\
057 & Motozintla & 782.50 \\
060 & Ocotepec & 59.60 \\
070 & El Porvenir & 121.70 \\
080 & Siltepec & 685.60 \\
& Total de la Región & 1897.90 \\
\hline REGION VALLES CENTRALES FRONTERIZOS \\
Municipios & Extensión en km² \\
\hline 006 & Amatenango de la Frontera & 171.40 \\
030 & Chicomuselo & 958.90 \\
034 & Frontera Comalapa & 717.90 \\
099 & La Trinitaria & 1840.70 \\
& Total de la Región & 3688.90 \\
\hline IV. REGION SELVA & \\
Municipios & Extensión en $\mathrm{km}^{2}$ \\
\hline 019 & Comitán de Domínguez & 1043.30 \\
041 & La Independencia & 1704.10 \\
052 & Las Margaritas & 5718.40 \\
059 & Ocosingo & 10691.60 \\
& Total de la Región & 19157.40 \\
\hline & &
\end{tabular}

Fuente para extensión: Dirección General de Estadística VIII Censo General de Población. Chiapas. Secretaría de Industria y Comercio, 1960. pp.25-27.

En la figura 1 se puede observar la localización de las regiones siguiendolos límites municipales. Las diferencias con la regionalización de Velasco $(1979)^{9}$ consisten en diferenciar el Soconusco en la región,

9 Jesús Agustín Velasco (1979) intentó llegar a nivel municipal para la definición de las regiones. Reconoció la existencia de siete regiones de la siguiente manera: 1) Región planicie costera. Soconusco; 2) Región Sierra Madre; 3) Región depresión central del Grijalva; 4) Región meseta central o Altos de Chiapas; 5) Región Norte del estado;6) Región Planicie del Golfo "Petrolera"; y, 7) Región selva Lacandona. Esta regionalización podría ser motivo de perfeccionamiento en la medida en que las 
introducir a Amatenango de la Frontera como parte de los valles centrales, hacer un recorte en la región de la depresión central señalando los municipios fronterizos exclusivamente ( $\mathrm{y}$ no toda la región que ocupa gran parte del estado), incluir en la selva al municipio de Comitán de Domínguez, envirtud de la gran importancia que ejerce en la zona fronteriza la ciudad homónima; y por último, excluir aAltamirano, en virtud de que noexiste ocupación de jornaleros guatemaltecos, ya que ocupa mano de obra de los Altos de Chiapas.

\section{Regiones fronterizas de Guatemala}

La detección de espacios territoriales donde existiera una relación homogénea entre los distintos procesos de trabajo fue el criterio que orientó la delimitación regional de Guatemala. De ahí que fuera indicado asumir la regionalización derivada de la actividad agrícola como la base para la organización espacial de la frontera.

\section{Regionalización agrícola de Guatemala}

A pesar de haberse realizado varios ensayos para regionalizar al país ${ }^{10}$, tiene especial relevancia el trabajo de Braudio Morán Burgos (1970), el cual es retomado para el desarrollo de este trabajo. Este autor establece la regionalización de Guatemala basándose en la agricultura, por ser la actividad económica principal.Tal estudio partió de un análisis biofísico y otro socioeconómico, ambos permitieron la agrupación de los municipios a través de la ponderación de las variables que componían la escala de calificación de éstos, de donde resultaron las siguientes regiones:

subdivisiones se hicieran con base en una tipificación por municipio de una serie de factores que debían ponderarse y de esta cuenta darle más base. No obstante, conviene traerla a cuenta porque después de ésta, las regionalizaciones de Chiapas han sido variantes de la geografía-física de Bassols o de la de Velasco.

10 Cfr. Secretaría General del Consejo de Planificación Económica. Regionalización preliminar del territorio de la República de Guatemala. Guatemala: s.e., 1966, donde se da noticia de las regionalizaciones de la Comisión Nacional del Salario, Aeronáutica Civil, Dirección General de Caminos, Dirección General de Servicios Agrícolas, etc. Instituto de Fomento Municipal INFOM Proyecto para la regionalización municipal. Guatemala: s.f., el estudio elaborado en 1966 es pionero en trabajar a nivel municipal para definir regiones. La utilización de polos de organización regional o de atracción fue realizada por obras públicas con magros resultados: Cfr. Departamento de Planeamiento Urbano y Regional. Política Nacional de Desarrollo Regional, Delimitación Regional. Guatemala: Obras Públicas, 1967. 
Regiones agrícolas de Guatemala

\begin{tabular}{|c|c|}
\hline Región & Subregión \\
\hline Altiplano occidental & $\begin{array}{l}\text { Altiplano Central } \\
\text { Cuchumatanes y Tierras } \\
\text { Bajas }\end{array}$ \\
\hline Ixcán-Chixoy & \\
\hline Pacífico occidental & $\begin{array}{l}\text { Pie de Monte Occidental } \\
\text { Planicie costera } \\
\text { Occidental }\end{array}$ \\
\hline Pacifico oriental & $\begin{array}{l}\text { Pie de Monte Oriental } \\
\text { Planicie costera } \\
\text { oriental }\end{array}$ \\
\hline $\begin{array}{l}\text { Valles y tierras altas } \\
\text { centrales }\end{array}$ & $\begin{array}{l}\text { Valle Motagua } \\
\text { Salamá } \\
\text { Central }\end{array}$ \\
\hline Oriental & $\begin{array}{l}\text { Llanuras y tierras } \\
\text { altas orientales } \\
\text { Valles Olopacamotán }\end{array}$ \\
\hline Polichic Caribe & \\
\hline Cobanera & $\begin{array}{l}\text { Sierras, valles y } \\
\text { colinas Cobaneras } \\
\text { Planicie cobanera }\end{array}$ \\
\hline $\begin{array}{l}\text { Pasión Sur Petén } \\
\text { Nor-Petén Itzá }\end{array}$ & $\begin{array}{l}\text { San Pedro Chocop } \\
\text { Lacandón } \\
\text { Tikal }\end{array}$ \\
\hline
\end{tabular}

\section{Definición de la regiones fronterizas de Guatemala}

Para definir las regiones fronterizas se parte del criterio de lindar con México; así, las subregiones I-2, II, III-1, III-2, IX y X-2 de la regionalización agrícola mencionada se encuentra en la frontera con México. En virtud de que la subregión I-1 es muy extensa se hizo un recorte en ella para efectos de la delimitación fronteriza, excluyéndose los departamentos de Sololá, Chimaltenango y Sacatepéquez; definiéndose la región I-1F o sea Altiplano Central Fronterizo como la parte de la subregión I-1 que colinda con la frontera mexicana. Las otras quedaron sin cambios, de acuerdo a la figura 1. 


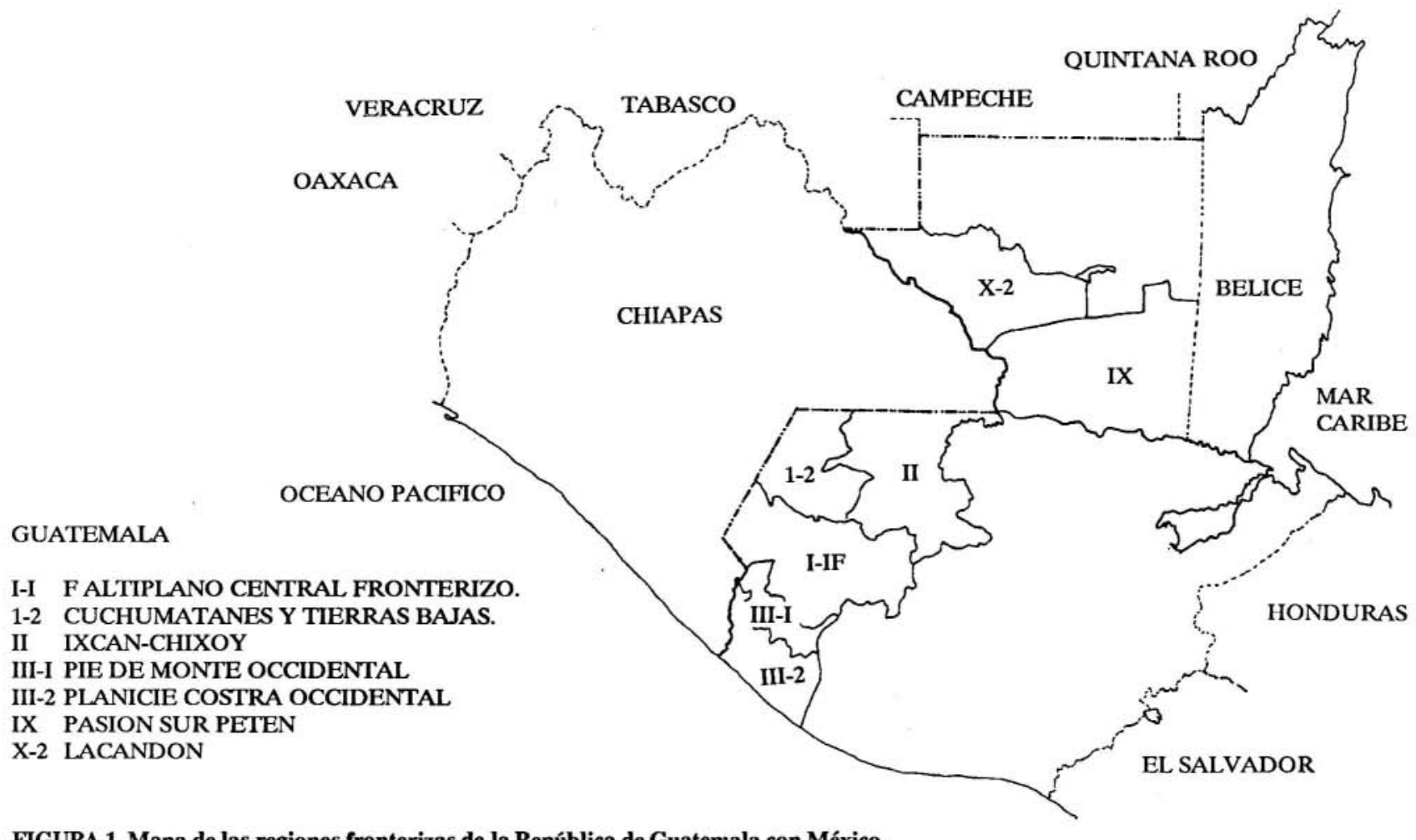

FIGURA 1. Mapa de las regiones fronterizas de la República de Guatemala con México. 


\section{LA MIGRACION DE JORNALEROS}

\section{Bases explicativas de la migración}

La tendencia favorable al crecimiento del flujo de jornaleros guatemaltecos no fue debida a las diferencias salariales entre las zonas cafetaleras de México y Guatemala, pues en este sentido formaban una unidad de oferta. Durante la década de los cincuenta, el jornal estuvo alrededor de 80 centavos de dólar norteamericano (en esos años igual a quetzales y equivalente a $\$ 10.00$ de México), en los sesenta fluctuó alrededor de los 1.25 de dólar EUA (similar en quetzales y aproximadamente $\$ 20.00$ en México).

A partir de 1960, los jornaleros guatemaltecos llegan a Chiapas en forma creciente a ofrecer su fuerza de trabajo, hasta convertirse en elcomponente principal de los trabajadores ${ }^{11}$. Esta tendencia, presente hoy en día, fue sancionada por los pocos años de bonanza petrolera en que los terratenientes del Soconusco y de la sierra de Chiapas pudieron incrementar las contrataciones, ya que la reserva de mano de obra era mayor en Guatemala debido a que su población creció más rápidamente que la de Chiapas, así como por estar sujeta a procesos de proletarización más intensos. En la figura 2 se pueden observar comparativamente, las tendencias del crecimiento de la población tanto del estado de Chiapas como de Guatemala.

El crecimientode la poblaciónguatemalteca sumado a los procesos político-sociales, contribuyó a que los jornaleros de ese país se convirtieran en el principal componente de los trabajadores en el Soconusco y Sierra para la cosecha de café.

\section{Referencias demográficas en las regiones fronterizas}

La frontera como demarcación constriñe la libre movilidad de las poblaciones agrupadas a ambos lados del linde. En consecuencia, en algunos momentos se producen desequilibrios en la aprobación territorial por encima de la continuidad espacial. Estos desequilibrios tienden a resolverse por la migración interregional e internacional.

${ }^{11}$ Impresión compartida por Vera K. de Hartwing (véase: Hartwing, 1980: 119). 


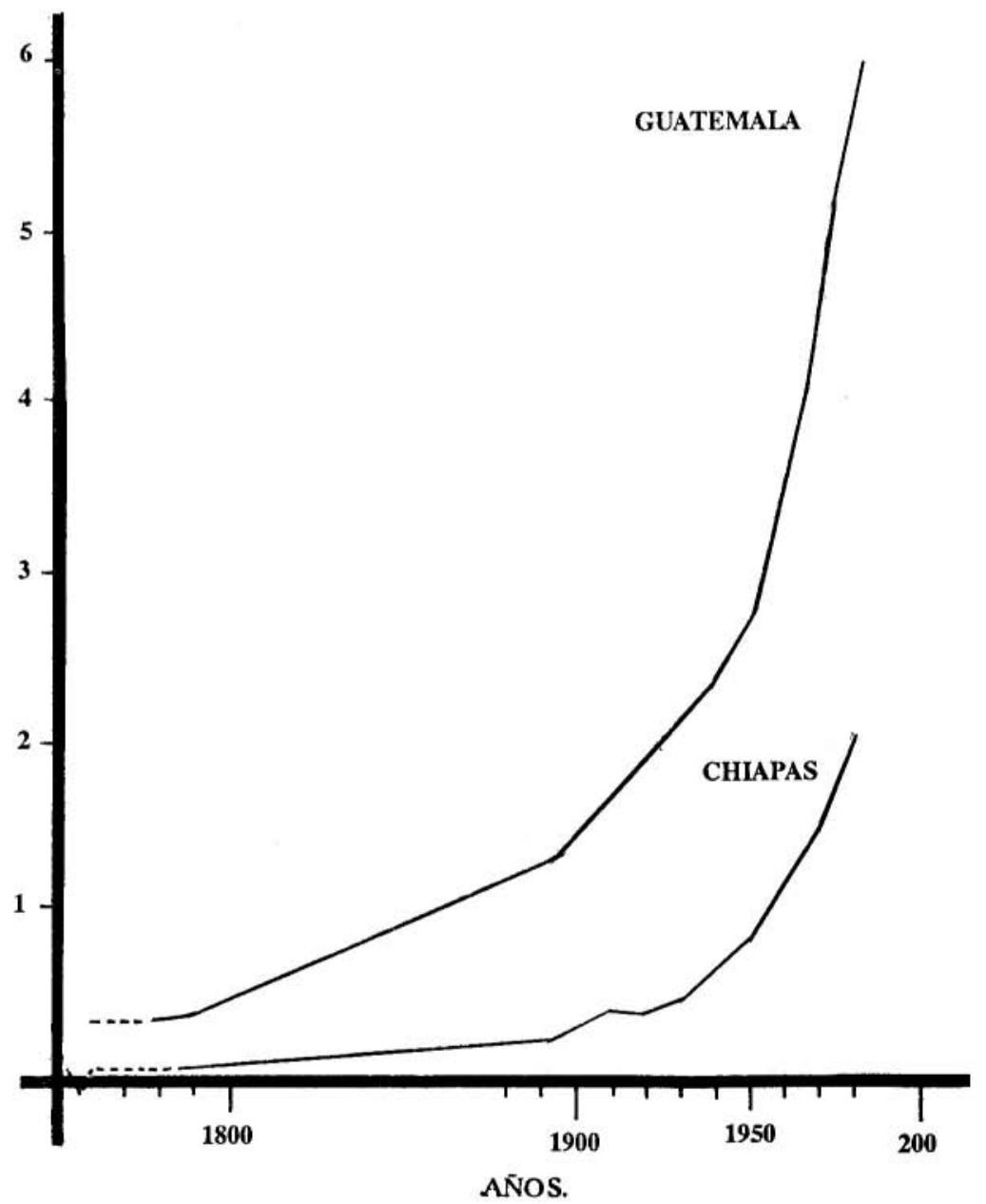

FIGURA 2. Evolución de la población en Chiapas y Guatemala. 
En términos relativos las poblaciones fronterizas de Chiapas y Guatemala comparten características semejantes. La edad mediana de la población es bastante similar como se muestra en el siguiente cuadro:

CUADRO 2. Promedio de edades en las regiones fronterizas de Chiapas y Guatemala.

\begin{tabular}{ccc|ccc}
\hline \multicolumn{3}{c|}{ C H I A P A S } & \multicolumn{3}{c}{ G U A T E M A L A } \\
\hline Región & \multirow{2}{*}{1970} & \multirow{2}{*}{1980} & Región & 1973 & 1981 \\
\hline Gran Región & 16.29 & 17.44 & Gran Región & 16.60 & 16.35 \\
& & & & & \\
Soconusco & 16.41 & \multirow{2}{*}{17.62} & III-1 & 16.85 & 16.31 \\
& & & III-2 & 16.43 & 16.62 \\
Sierra & 15.90 & \multirow{2}{*}{16.33} & I-1F & 16.83 & 16.66 \\
& & & & & \\
Valles & \multirow{2}{*}{15.93} & \multirow{2}{*}{16.81} & I-2 & 16.40 & 16.04 \\
Centrales & & \multirow{2}{*}{15.90} & II & 16.07 & 15.37 \\
Selva & 16.51 & 17.90 & IX & 15.20 & 14.76 \\
& & & X-2 & 16.37 & 15.92 \\
\hline
\end{tabular}

Aunque muy semejantes, aparece una diferencia en el sentido de que la población chiapaneca fronteriza se envejece mientras la guatemalteca se rejuvenece; naturalmente todavía no se puede hablar de una tendencia, sino apenas un indicio de una dinámica poblacional diversa.

El índice de la población masculina muestra igual semejanza a nivel de las regiones: 
CUADRO 3. Indice de la población masculina en las regiones fronterizas chiapaneca y guatemalteca.

\begin{tabular}{|c|c|c|c|c|c|}
\hline \multicolumn{3}{|c|}{ CHIA PAS } & \multicolumn{3}{|c|}{ G UA T E M A L A } \\
\hline Región & 1970 & 1980 & Región & 1973 & 1981 \\
\hline Gran Región & 104.80 & 102.50 & Gran Región & 102.99 & 101.07 \\
\hline Soconusco & 104.54 & 101.80 & $\begin{array}{l}\text { III-1 } \\
\text { III-2 }\end{array}$ & $\begin{array}{l}108.49 \\
106.07\end{array}$ & $\begin{array}{l}104.02 \\
102.42\end{array}$ \\
\hline Sierra & 111.70 & 106.65 & $\mathrm{I}-1 \mathrm{~F}$ & 99.87 & 98.28 \\
\hline $\begin{array}{l}\text { Valles } \\
\text { Centrales }\end{array}$ & 107.07 & 103.63 & I-2 & 103.20 & 102.51 \\
\hline Selva & 100.29 & 101.47 & II & 103.22 & 103.31 \\
\hline
\end{tabular}

Fuente: Chiapas: Dirección General de Estadística. Séptimo Censo General de Población. Chiapas. México: DGE, Secretaría de Economía, 1952. Dirección General de Estadística. VIIICenso General de Población. Chiapas. México: DGE, Secretaría de Industria y Comercio, 1960. Dirección General de Estadística. IX Censo General de Población. Chiapas. México: DGE, Secretaría de Industria y Comercio, 1971. Instituto Nacional de Estadística, Geografía e Informática. $X$ Censo General de Población y Vivienda, 1980. México: INEGI, Secretaría de Programación y Presupuesto, 1983. Guatemala: Instituto Nacional de Estadística. IX Censo General de Población de 1981. Guatemala: INE, 1985

El paso de las clases en edad infantil en general a este índice, no obstante, es interesante señalar que la región I-1F, altiplano central fronterizo, muestra el índice más bajo entre las regiones; si este índice está afectado por los patrones de mortalidad que suponemos similares para las regiones, es claro que el patrón de migración puede estar interviniendo para este caso.

A continuación se muestra el número de dependientes económicos de la población fronteriza, esto es, de cada 100 personas en edad de trabajar (de 15 a 64 años) se señala el total de éstas que tienen dependientes, en este caso niños y ancianos. 
CUADRO 4. Comparativo del número de niños y ancianos dependientes en las regiones fronterizas por cada 100 hab. en edad de trabajar.

\begin{tabular}{|c|c|c|c|c|c|}
\hline \multicolumn{3}{|c|}{ CHIA PAS } & \multicolumn{3}{|c|}{ G UA TEMALA } \\
\hline Región & 1970 & 1980 & Región & 1973 & 1981 \\
\hline Gran Región & $\begin{array}{r}95.02 \\
6.13\end{array}$ & $\begin{array}{r}87.39 \\
5.96\end{array}$ & Gran Región & $\begin{array}{r}92.02 \\
5.43\end{array}$ & $\begin{array}{r}94.40 \\
5.64\end{array}$ \\
\hline Soconusco & $\begin{array}{r}94.08 \\
6.13\end{array}$ & $\begin{array}{r}84.73 \\
6.06\end{array}$ & $\begin{array}{l}\text { III-1 } \\
\text { III-2 }\end{array}$ & $\begin{array}{r}90.70 \\
5.51 \\
\\
92.90 \\
5.02\end{array}$ & $\begin{array}{r}95.70 \\
6.17 \\
\\
92.02 \\
5.77\end{array}$ \\
\hline Sierra & $\begin{array}{r}97.49 \\
5.60\end{array}$ & $\begin{array}{r}95.48 \\
5.70\end{array}$ & $\mathrm{I}-1 \mathrm{~F}$ & $\begin{array}{r}90.39 \\
5.80\end{array}$ & $\begin{array}{r}91.94 \\
5.92\end{array}$ \\
\hline $\begin{array}{l}\text { Valles } \\
\text { Centrales }\end{array}$ & $\begin{array}{r}98.30 \\
5.75\end{array}$ & $\begin{array}{r}90.84 \\
5.33\end{array}$ & $\mathrm{I}-2$ & $\begin{array}{r}93.60 \\
5.27\end{array}$ & $\begin{array}{r}96.62 \\
5.31\end{array}$ \\
\hline Selva & $\begin{array}{r}94.06 \\
6.63\end{array}$ & $\begin{array}{r}87.66 \\
6.18\end{array}$ & II & $\begin{array}{r}96.03 \\
4.87\end{array}$ & $\begin{array}{r}101.74 \\
4.73\end{array}$ \\
\hline & & & IX & $\begin{array}{r}101.23 \\
2.95\end{array}$ & $\begin{array}{r}105.82 \\
3.19\end{array}$ \\
\hline & & & $\mathrm{X}-2$ & $\begin{array}{r}93.86 \\
5.61\end{array}$ & $\begin{array}{r}97.07 \\
4.85\end{array}$ \\
\hline
\end{tabular}

- De cada región la cantidad superior corresponde en niños y la inferior a los ancianos. liuente:Chiapas: Dirección General de Estadística. VII Censo General de Población. ( 'hiapas. México: DGE, Secretaría de Economía, 1952. Dirección General de I'sladística. VIII Censo General de Población. Chiapas. México: DGE, Secretaría de Industria y Comercio, 1960. Dirección General de Estadística. IX Censo General il' Población. Chiapas. México: DGE, Secretaría de Industria y Comercio. 1971. Instituto Nacional de Estadística, Geografía e Informática. $X$ Censo General de I'oblación y Vivienda, 1980. Chiapas. México: INEGI, Secretaría de Programación y I'resupuesto, 1983. Guatemala: Instituto Nacional de Estadística. IX Censo General de I'oblación de 1981. Guatemala: INE, 1985. 
Una visión general de la estructura de población se encuentra en la figura 3 , donde se dibujan las pirámides de edad.

CUADRO 3. Hipotesis de balance de fuerza de trabajo de Guatemala y los altos en el Soconusco y la sierra de Chiapas.

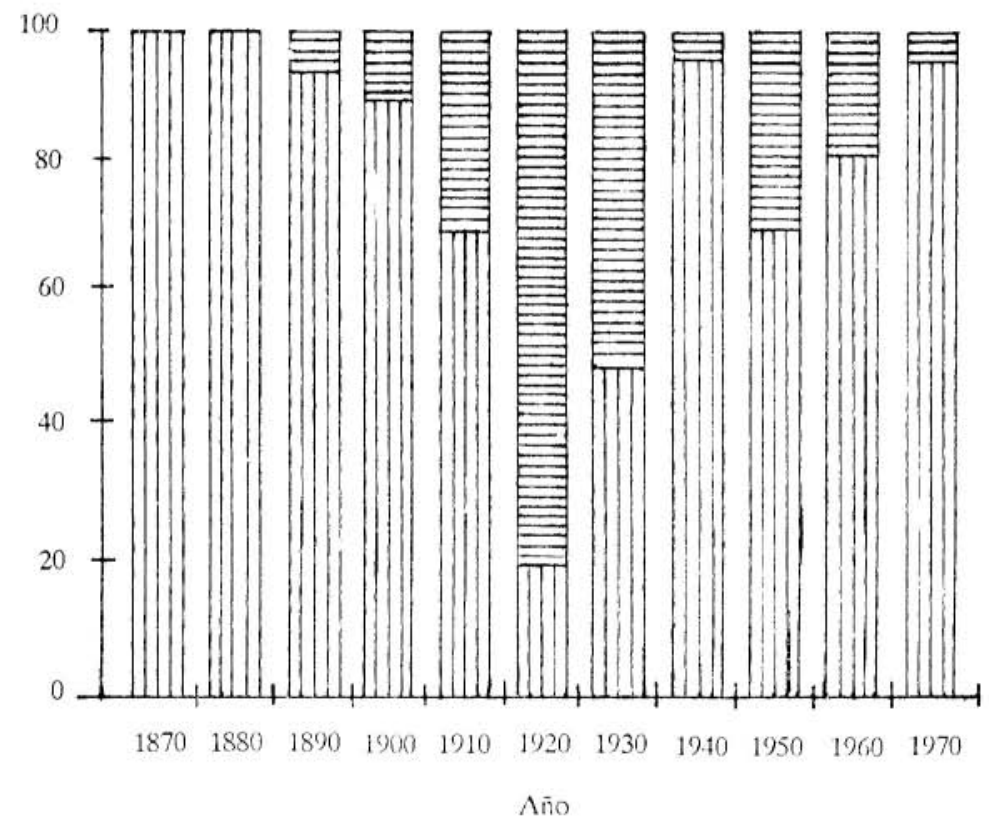
Altos de Chiapas
Guatemaltecos 
Como se observa, a pesar de que las pirámides son expansivas, lo que indica la gran similitud de ambas poblaciones, la de Chiapas de 1980 denota un movimiento hacia la construcción de la población al reducir la clase de 0 hasta cuatro años en el porcentaje sobre el total. Esto podría indicar el comienzo de una dinámica diferente de crecimiento poblacional entre ambas regiones fronterizas; naturalmente que tal tendencia tendría que mantenerse por varios decenios para llegar a poderse afirmar este hecho.

Conviene tomar en cuenta la densidad de población como un elemento explicativo de la migración internacional. El siguiente cuadro enfrenta el dato de habitantes por kilómetro cuadrado:

CUADRO 5. Densidad de la población en las regiones fronterizas de Chiapas y Guatemala (habitantes por kilómetro cuadrado).

\begin{tabular}{|c|c|c|c|c|c|}
\hline \multicolumn{3}{|c|}{ CHIAPAS } & \multicolumn{3}{|c|}{ G UA TEM A LA } \\
\hline Región & 1970 & 1980 & Región & 1973 & 1981 \\
\hline Gran Región & 18.37 & 25.03 & Gran Región & 39.22 & 47.12 \\
\hline Soconusco & 52.16 & 68.55 & $\begin{array}{l}\text { III-1 } \\
\text { III-2 }\end{array}$ & $\begin{array}{r}131.66 \\
72.73\end{array}$ & $\begin{array}{r}154.14 \\
86.76\end{array}$ \\
\hline Sierra & 37.44 & 47.34 & $\mathrm{I}-1 \mathrm{~F}$ & 109.01 & 131.21 \\
\hline $\begin{array}{c}\text { Valles } \\
\text { Centrales }\end{array}$ & 18.79 & 25.42 & $\mathrm{I}-2$ & 51.05 & 59.55 \\
\hline Selva & 6.21 & 9.63 & $\begin{array}{c}\text { II } \\
\text { IX } \\
X-2\end{array}$ & $\begin{array}{r}23.31 \\
3.29 \\
1.25\end{array}$ & $\begin{array}{r}24.45 \\
6.49 \\
3.21\end{array}$ \\
\hline
\end{tabular}

Este cuadro es muy revelador si tenemos en cuenta que en Chiapas la población económicamente activa se emplea en un $57.42 \%$ en la 
agricultura y en Guatemala en un $53.95 \%{ }^{12}$, de lo que resulta que la relación hombre-tierra es bastante significativa. Los datos muestran que la gran región fronteriza de Guatemala tiene una densidad de casi el doble de la chiapaneca. La forma como se distribuye en las regiones esta mayoría es sugerente: la región cafetalera de Guatemala (III-1 Pie de Monte Occidental) tiene la más alta densidad de todas las regiones, mientras que en Chiapas, el Soconusco y la sierra, donde una parte de su territorio se emplea para estos fines, tienen una densidad bastante baja en relación a aquélla; el antiplano central fronterizo de Guatemala $\mathrm{I}-1 \mathrm{~F}$, zona de donde proviene gran parte de la fuerza de trabajo estacional de Guatemala, tiene una densidad que no es superada por ninguna de la regiones fronterizas de Chiapas. Sólo en la selva, Chiapas tiene una densidad superior a las regiones homólogas de Guatemala: II Ixcán-Chixoy, IX Pasión Sur Petén y X-2 Lacandón.

La densidad se convierte así en la base necesaria para la explicación del movimiento migratorio. Es conocido que la expansión de cultivos siempre va a encontrar más fácilmente trabajadores en la regiones más pobladas. También allí, los procesos de proletarización van a ser más intensos, pues la frontera agrícola no se puede expandir tan fácilmente como en los sitios menos poblados, es decir, que la economía campesina, para su supervivencia, necesita del trabajo asalariado que posibilite el uso de tierras marginales.

\section{CONCLUSION}

Debido a la gran relación que existe entre las poblaciones do Chiapas y Guatemala, ha surgido la necesidad de su estudio. En su inicio, esta tarea no ha sido fácil, ya que es necesario despojarla do intereses ideológicos, mismos que se manifestaron en un vivo debate. $\mathrm{Al}$ conocer las corrientes migratorias debemos situar el ámbito del problema haciendo una regionalización que ubique geográficamento el fenómeno. La aceptación de las regionesfronterizas que se proponen en este estudio ayudará en el acopio futuro de datos.

${ }^{12} \mathrm{Cfr}$. Censos de población citados. Chiapas: PEA: 734047 empleados en elagro: 421561 de los cuales son patrones: 10911 . Guatemala: PEA: 1683828 empleados en el agro: 908513 de los cuales son patrones 11 551. Los datos no soa comparablen estrictamente, pues en Guatemala se cuenta desde diez años para arriba, mientras c\| Chiapas de 12 en adelante. 
El marco regional debe trabajarse a nivel histórico y a nivel demográfico, pues en ellos se expresa la base material que permitirá la explicación general de los procesos de integración territorial. En efecto, el sureste de México experimenta un fuerte movimiento hacia constituir un continuum económico con las regiones centroamericanas vecinas. La conciencia de este movimiento social ayudará en la generación de políticas que tiendan al desarrollo económico, a la armonía social entre los pueblos de distintos países y al entendimiento de tradiciones culturales que si bien no son disímiles, sí se han manifestado en desarrollos separados. 


\section{B I B L I O G R A F I A}

AGUAYO Q., Sergio. 1984. "La línea móvil del sur". En: Nexos No. 79. México. Año VII, Vol. 7, julio 1984.

AGUAYO Q., Sergio. 1985a. El éxodo centroamericano: consecuencias de un conflicto. México, Secretaría de Educación Pública.

AGUAYO Q., Sergio. 1985b. "Exodo centroamericano". En: Nexos No. 88. México, Año VIII, Vol. 8 Abril 1985.

ALBA, Francisco, 1984. La población de México: evolución y dilemas México. El Colegio de México.

BARRIOS Ruiz, Walda. 1976. El Derecho de asilo. Tesis de la Facultad de Ciencias Jurídicas y Sociales. Universidad de Sar Carlos de Guatemala. Guatemala.

BLEJER, Mario I., et al. 1982. "Un análisis de los determinantes económicos de la migración mexicana legal e ilegal hacia los Estados Unidos". En Mina, Alejandro (comp.) Lecturas sobre temas demográficos. México, El Colegio de México.

HARTWIG, Vera K. 1980. "Procesos de integración indígena en la producción agraria y artesanal en Chiapas y Tabasco". En: varios autores. El sur de México. Datos sobre la problemática indígena. México, Dirección General de Publicaciones. UNAM.

MAYORGA, Pedro. 1984. "Los refugiados, entre Ixcán y Televisa (ur tema que nos interesa a todos)". En Sábado Suplementc cultural de unomásuno. México, 4 de agosto.

MORA Bravo, Miguel. 1984. El derecho de la planeación familiar. México. CONAPO.

MORAN Burgos, Braudio L. 1970. Regionalización agrícola de Guatemala. Programa SIBCA/IICA, Guatemala. Consejo de Planificación Económica.

MOSQUERA Aguilar, Antonio. 1978. La región oriental de Guatemala. Centro Universitario de Oriente de la Universidad de San Carlos de Guatemala (mimeo).

POHLENZ Córdova, Juan. 1978. Las formaciones de las plantaciones cafetaleras de Soconusco y el capitalismo en Chiapas. San Cristóbal de Las Casas: CIES. (mimeo).

POHLENZ C., Juan y Ma. Teresa Castillo B. 1982. Fuerza de trabajo y producción agrícola en Soconusco, Chiapas. San Cristóbal de Las Casas: C.L.C. CIES. (mimeo). 
TABBARAH, Riad. 1982. "Migración internacional de los países menos desarrollados a los países más desarrollados: soluciones y políticas". En: Urquidi, Víctor L. y José B. Morelos (comps.). Tendencias y políticas de población. México, El Colegio de México.

TUIRAN, Rodolfo. 1983. "Estimaciones sobre el tamaño de la población indocumentada en los Estados Unidos". En: Curso sobre aspectos básicos del fenómeno demográfico. Xalapa, Ver. AMEP.

VELASCO S., Jesús Agustín. 1979. El desarrollo comunitario de la Sierra Madre de Chiapas. México. UNAM.

WASSERSTROM, Roberto. 1976. "El bracerismo guatemalteco en Chiapas ¿Un motivo de orgullo para el pueblo mexicano?”. En: Punto crítico, México, México, Año V \#62. 